\title{
Modeling and Control of a Flying Robot for Contact Inspection
}

\author{
M. Fumagalli, R. Naldi, A. Macchelli, R. Carloni, S. Stramigioli and L. Marconi
}

\begin{abstract}
This paper focuses on the modeling and control of a flying robot. The complete system, composed of a quadrotor unmanned aerial vehicle and a custom-made manipulator, has been designed for remote inspection by contact of industrial plants. The goal of this paper is to show the dynamical characteristics of the flying robot during tasks that require physical interaction, and to determine a control strategy that allows to safely interact with unknown environments. The methodology has been implemented on a real prototype and tested in an indoor area. Experimental results validate the proposed controller and show its effectiveness.
\end{abstract}

\section{INTRODUCTION}

Physical interaction between robotic devices and their surrounding environment is a research trend that is spread among different disciplines. A major point of interest lays in endowing these devices with autonomous behavior, and with the capability of safely performing actions and interaction in unstructured environments, without necessarily requiring human skills, or human presence. Unmanned Aerial Vehicles (UAVs) are a sub-category of aerial vehicles that do not carry a human operator. These vehicles can fly autonomously or be piloted remotely. Among this category, autonomous rotorcrafts stand out for their characteristics. Due to their dexterity and small dimensions, in fact, they can fly in both open spaces and closed environments [1], [2].

Nevertheless, the tasks that an autonomous flying system can carry out are still limited, especially in real applicative scenarios. The main challenge is to create novel robotic platforms that are not only capable of performing tasks that require autonomous behaviors, but also to physically interact with remote environments. To create such autonomous systems, some methodological issues should first be addressed. As an example, the actuation of most of these flying vehicles, does not allow to simultaneously actuate all the degrees of freedom (DOFs) of the floating base platforms. Among the different aerial configurations, quadrotors [3], [4], helicopters [5] or ducted-fans [6], are indeed under-actuated mechanical systems [7]. When defining a control law for these systems, the dynamical properties should be taken into account, and stability should be assured not only during free-flight, but also when interaction with the surrounding environment occurs. Research on this topic have been conducted in [8],

\footnotetext{
*This work has been funded by the European Commission's Seventh Framework program as part of the project AIRobots under grant no. 248669.

M. Fumagalli, R. Carloni and S. Stramigioli are with the Faculty of Electrical Engineering, Mathematics and Computer Science, University of Twente, The Netherlands. \{m.fumagalli, r.carloni, s.stramigioli\}@utwente.nl

R. Naldi, A. Macchelli and L. Marconi, are with the Faculty of Engineering, University of Bologna, Italy. Email: \{roberto.naldi, alessandro.macchelli, lorenzo.marconi \}@unibo.it
}

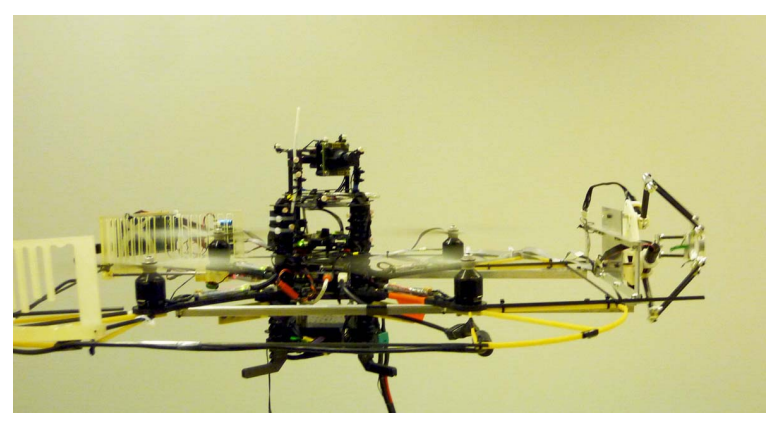

Fig. 1. AscTec Pelican quadrotor hovering with a manipulator on its side

where a quadrotor helicopter is employed to clean a surface while hovering. In this case, the under-actuation issue is addressed by employing an additional propeller, to counteract contact forces while maintaining the stability of the vehicle.

In [9], multiple quadrotors cooperate to perform grasping and transportation of objects, while in [10] they are exploited to assembly an infrastructure. The control of aerobatic airplanes is addressed in [11], [12], to perform landing on a vertical surface. The stability of the vehicle during the interaction with a compliant environment has been studied in [13], where an autonomous helicopter endowed with a manipulator is considered, while aerial manipulation using an aerial system has been investigated in [14]. Similarly, the European project AIRobots [15] has the goal of developing a new generation of service robots, able to actively support the human operators in performing tasks in remote or unsafe environments.

In this work, a quadrotor aerial vehicle endowed with a manipulation system is studied. The paper addresses the dynamics and control of the overall under-actuated flying robot, i.e., the aerial manipulator, during interaction tasks. The manipulation system, mounted on the quadrotor UAV has been designed to be the hand of the aerial manipulator. It will be shown hereafter that, through the end-effector of the manipulation system, the aerial vehicle is capable of performing interaction tasks during flight.

The paper is organized as follows: Sec. II shows the dynamic modeling of the flying robot in interaction with the environment. Sec. III presents the control strategy together with a proof of the passivity of the controlled system. In Sec. IV, experiments validate that the flying robot is capable to safely interact with the environment by means of the manipulation system's end-effector. 


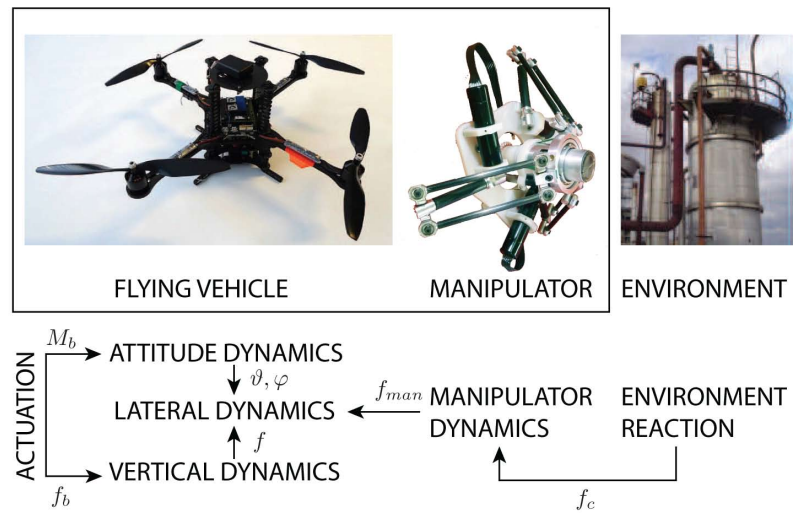

Fig. 2. The aerial manipulator is composed by the quadrotor and the manipulator in interaction with the environment. The different parts of the system exchange forces during the interaction.

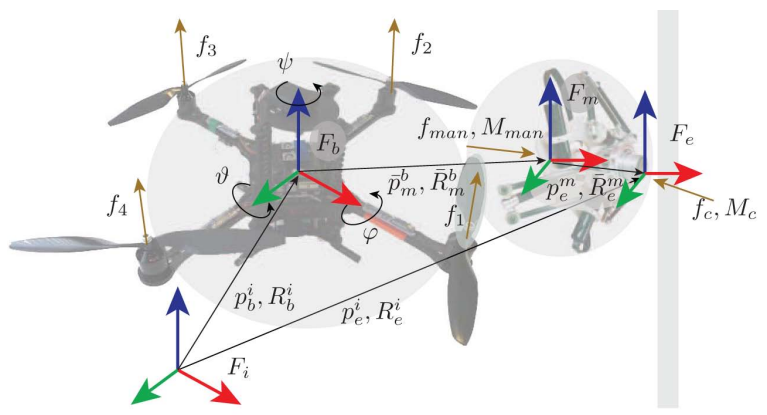

Fig. 3. Kinematic notation of the overall system. $F_{i}$ indicates the inertial frame, $F_{b}$ the center of gravity of the UAV, $F_{m}$ the base of the manipulation system, $F_{e}$ the end-effector reference frame.

\section{SYSTEM MODEL}

An analysis of the overall dynamical model of the system, composed of a quadrotor UAV mounting a manipulator on a side and the environment (see Fig. 2), is required to address the control and the stability of the under-actuated aerial manipulator. The plant is capable of both performing free flight and interacting with the remote environment by means of the end-effector of the manipulation system. The advantages of such kind of system is not only that the overall manipulation workspace becomes ideally unbounded, but also that, through a proper kinematic structure of the manipulation system, the problems related to the underactuation, typical of most UAV vehicles, is reduced in tasks such as precise point tracking.

The overall dynamics can thus be decomposed into three main subsystems, i.e. the aerial vehicle, the manipulation system and the environment, interacting together at specific interconnection points along a floating base kinematic structure. The coupled model of the quadrotor and the manipulator is exploited to analyze the effects on the vehicle dynamics caused by the manipulation system interacting with the environment. Through this analysis, we define a control law able to passify the aerial manipulator during interaction tasks.

\section{A. Notation}

With reference to Fig. 3, the kinematic notation is:

- $F_{i}, F_{b}, F_{m}$ and $F_{e}$, the inertial frame, the body frame having the center of gravity (c.g.) of the UAV as origin, the reference frame attached to the base plate of the manipulation system, and the end-effector frame, respectively;

- $p_{b}^{i}=\left[x_{b}^{i}, y_{b}^{i}, z_{b}^{i}\right]^{T}$ and $R_{b}^{i} \in \mathbb{R}^{3 \times 3}$ the position and rotation matrix of the c.g. of the UAV with respect to the inertial frame $F_{i}$;

- $\bar{p}_{m}^{b}=\left[\bar{x}_{m}^{b}, \bar{y}_{m}^{b}, \bar{z}_{m}^{b}\right]^{T}$ and $\bar{R}_{m}^{b} \in \mathbb{R}^{3 \times 3}$ the constant position and rotation matrix of the base of the manipulation system with respect to the UAV's c.g., i.e., $F_{b}$;

- $p_{e}^{m}=\left[x_{e}^{m}, y_{e}^{m}, z_{e}^{m}\right]^{T}$ and $R_{e}^{m} \in \mathbb{R}^{3 \times 3}$ the position and rotation matrix of the end-effector of the manipulation system with respect to its base frame $F_{m}$;

- $p_{e}^{i}=\left[x_{e}^{i}, y_{e}^{i}, z_{e}^{i}\right]^{T}$ and $R_{e}^{i} \in \mathbb{R}^{3 \times 3}$ the position and rotation matrix of the end-effector of the manipulation system with respect to the inertial frame $F_{i}$.

The dynamical terms are:

- $f_{b} \in \mathbb{R}$ the total thrust on the UAV generated by the propellers; $f_{\text {man }}^{m} \in \mathbb{R}^{3}$ the reaction force the manipulator and the UAV reciprocally exchange at the manipulator base;

- $M^{b}=\left[M_{x}, M_{y}, M_{z}\right]^{T} \in \mathbb{R}^{3}$ the control torque of the UAV; $M_{g y}$ is the moment vector, which denotes the gyroscopic effects due to the rotating propellers. $M_{\text {man }}^{m} \in \mathbb{R}^{3}$ the reaction torque the manipulator and the UAV reciprocally exchange at the manipulator base;

- $f_{c}, M_{c} \in \mathbb{R}^{3}$ the force and moment vectors applied by the environment to the end-effector;

- $m_{\text {uav }}, J_{\text {uav }}$ are the UAV's mass and the inertia matrix;

- $f_{I}^{m} \in \mathbb{R}^{3}$ the vector of all the dynamical forces (inertial, centrifugal and Coriolis, and gravitational components) due to the absolute motion of the manipulator, in $F_{m}$;

- $M_{I}^{m} \in \mathbb{R}^{3}$ the vector of all the inertial, centrifugal and Coriolis, and gravitational components due to the absolute motion of the manipulator, in $F_{m}$;

- $g$ denotes the gravity acceleration.

To better describe the dynamics of the flying robot, the interaction between the aerial vehicle, the manipulator and the environment are captured by means of separated dynamical systems, interconnected together at specific points through localized interaction forces and moments. As shown in Fig. 3, the flying robot comes in interaction with the environment at the end-effector frame $F_{e}$, while the latter is rigidly connected, through its base, to the aerial vehicle at frame $F_{m}$. The frame $F_{b}$ of the UAV is not constrained, thus it is free to float with respect to the inertial frame $F_{i}$, due to the actuation forces generated by the propellers.

Note that the flying robot is an under-actuated mechanical system with a total of $n+6$ DOFs and $n+4$ control inputs [7], [16], [17]. The former are given by the lateral/longitudinal position ( $3 \mathrm{DOFs}$ ), the attitude ( $3 \mathrm{DOFs}$ ) of the UAV, by the position of the manipulator ( $n$ DOFs) [18]; the latter are 
the four forces produced by the four propellers and the $n$ actuation forces governing the robotic arm.

\section{B. Quadrotor}

With reference to the stand alone quadrotor UAV, note that by varying the relative thrust of the propellers, a net torque can be applied in any direction. The mapping of the forces generated by the four propellers $\left(f_{i}, i=1, \cdots, 4\right)$ into the total thrust and body moment $\mathbf{M}^{b}=\left[M_{x}, M_{y}, M_{z}\right]^{T}$ is given by the linear and constant relation [19]:

$$
\left[\begin{array}{c}
f_{b} \\
M_{x} \\
M_{y} \\
M_{z}
\end{array}\right]=\left[\begin{array}{cccc}
1 & 1 & 1 & 1 \\
0 & -d & 0 & d \\
d & 0 & -d & 0 \\
-c & c & -c & c
\end{array}\right]\left[\begin{array}{l}
f_{1} \\
f_{2} \\
f_{3} \\
f_{4}
\end{array}\right]
$$

where $d$ represents the distance from the UAV center of mass to the propeller, and $c$ the constant ratio between the propeller reaction torque and the generated thrust. The UAV is assumed to be described by a single rigid body with control force mappings to actuators and forces and moments exchanged with the manipulation system. The equations describing the dynamics of a quadrotor UAV are:

$$
\left\{\begin{aligned}
m_{u a v} \dot{\mathbf{v}}^{i}= & m_{u a v} g \hat{\mathbf{z}}^{i}+f R_{b}^{i}[0,0,-1]^{T}+R_{b}^{i} R_{m}^{b} f_{m a n}^{m} \\
\mathbf{J}_{\mathbf{u a v}} \dot{\boldsymbol{\omega}}_{b}^{b, i}=- & \boldsymbol{\omega}_{b}^{b, i} \times \mathbf{J}_{\mathbf{u a v}} \boldsymbol{\omega}_{b}^{b, i}+\mathbf{M}_{g y}+\mathbf{M}^{b}+ \\
& +R_{b}^{i} R_{m}^{b} M_{\text {man }}^{m}+R_{b}^{i}\left(R_{m}^{b} f_{\text {man }}^{m} \times p_{m}^{b}\right)
\end{aligned}\right.
$$

where $\boldsymbol{\omega}_{b}^{b, i}$ and $\dot{\omega}_{b}^{b, i}$ are the rotational velocity and acceleration, respectively, of the UAV with respect to the inertial frame, expressed in $F^{b} \cdot \mathbf{v}^{i}=\dot{p}_{b}^{i}$ and $\dot{\mathbf{v}}^{i}=\ddot{p}_{b}^{i}$ the linear velocity and acceleration of the UAV's c.g. in the inertial frame. The control forces are given by the total thrust $f_{b}$ and the control torque $\mathbf{M}^{b}$, as in Eq. 1. Note that the manipulator introduces a dynamical contribution to the UAV's dynamics in terms of forces $f_{\text {man }}$ and moments $M_{\text {man }}$ that act at the interconnection between the flying vehicle and the manipulator at $F_{m}$.

\section{Manipulation System}

The manipulator's dynamics can be described by means of the dynamical contribution of the internal dynamics ${ }^{1}$ of the manipulator itself (i.e. inertial, centrifugal, Coriolis and gravitational components) and the forces $f_{c}$ and moments $M_{c}$ that arise from the contact of the end-effector with the environment. The generalized forces at the manipulator's base can be considered as the actuation forces of the structure, i.e., the forces necessary to move the overall manipulator's mass. On the other hand, these generalized forces give origin to the reaction forces and moments on the UAV, which are disturbances to the UAV dynamics. From the equilibrium of dynamical forces and moment of the manipulator, the dynamical effect of a manipulation system acting on the

\footnotetext{
${ }^{1}$ The forces/moments at the manipulator's base, due to the manipulator's dynamics, depend on the manipulator's state (i.e. joints' position, velocity and acceleration) and on the UAV's linear/angular velocity and acceleration.
}

quadrotor at frame $F_{m}$ are described by:

$$
\left\{\begin{aligned}
f_{\text {man }}^{m} & =f_{I}^{m}+R_{e}^{m} f_{c}^{e} \\
M_{\text {man }}^{m} & =M_{I}^{m}+R_{e}^{m} M_{c}^{e}+R_{e}^{m} f_{c}^{e} \times p_{e}^{m}
\end{aligned}\right.
$$

Note that the forces and torques transmitted to the quadrotor at $F_{m}$, through the interconnection between the manipulator base and the UAV at $\bar{p}_{m}^{b}$ with respect to $F_{b}$, are the generalized forces due to the interaction and the forces and torques that arise from the manipulator's dynamics.

\section{Environment}

The environment is considered as a compliant surface at a certain location in the inertial frame $F_{i}$. We assume that the flying robot interacts with this surface at a point $\bar{p}_{i}$ with the end-effector, whose position is described by the point $p_{e}$ in the inertial frame $F_{i}$. The dynamic of the interaction can be described by adopting a Hunt-Crossley interaction model [20], where the penetration variable $x$ is given by $x=p_{e}-\bar{p}_{i}$. When the position $p_{e}$ of the end-effector is greater or equal than the position of the surface, the environment applies to the end-effector a force $f_{c}$ directed along the opposite direction of the penetration $x$. In other words, if two bodies collide, the normal reaction force is dependent on the penetration-depth $x$ ans is given by:

$$
f_{c}^{n}(x)= \begin{cases}k x^{n}(t)+\lambda x^{n}(t) \dot{x}(t) & x<0 \\ 0 & x \geq 0\end{cases}
$$

\section{CONTROL ARCHITECTURE}

The aerial manipulator differs from classical robotic systems [21], mainly because forces and momenta that arise from interaction cannot be completely rejected by the constraints at the base of the aerial manipulator. It follows that the actuators of such mechanism should provide the necessary reaction forces, to counteract those disturbances.

\section{A. Control Assumptions}

In this section, it is shown how to derive a control law that acts on the actuators of the under-actuated floating base in order to generate the desired reaction forces on the body frame of the aerial vehicle. By using a reduced order dynamical model, where the vehicle attitude $\Theta=$ $[\varphi, \vartheta, \psi]^{T}$ is employed as a virtual input, the aerial manipulator becomes capable of applying forces not only in the vertical direction but also in the lateral and longitudinal ones, meaning that the aerial vehicle is capable of counteracting the disturbance force $f_{\text {man }}$. The virtual input $\Theta$ directly affects the position of the end-effector in the inertial reference frame. As a consequence, during free-flight, tracking performances of the position of the end-effector may be poor if large reaction forces have to be compensated due to the motion of the end-effector itself. Nevertheless, this problem might be minimized by properly designing the manipulation system so as to have the mass of all moving parts sufficiently smaller than the one of the UAV, and, in turn, to reduce the influence of $f_{I}^{m}$ and $M_{I}^{m}$ on the aerial vehicle dynamics. 
To support the proposed cascade control approach, a highgain attitude control is required [22] in order to compensate for the reaction forces $f_{m a n}$ and torques $M_{\text {man }}$, which affect the attitude subsystem both in free-flight and during physical interaction. With these assumptions, the system dynamics can be reformulated as a reduced order dynamical system, i.e.,

$$
\left\{\begin{aligned}
m_{\text {uav }} \dot{\mathbf{v}}^{i} & =m_{\text {uav }} g \hat{\mathbf{z}}^{i}+f R_{b}^{i}[0,0,-1]^{T}+R_{b}^{i} R_{m}^{b} f_{\text {man }}^{m} \\
f_{\text {man }}^{m} & =f_{I}^{m}+R_{e}^{m} f_{c}^{e}
\end{aligned}\right.
$$

where the control inputs are $u=[f, \varphi, \vartheta, \psi]^{T}$, i.e. the thrust force $f$ and the attitude of the quadrotor.

Note that the orientation of the body frame $F_{b}$ with respect to the inertial frame $F_{i}$ can be defined by a rotation matrix $R_{b}^{i}$, that is function of the attitude $\Theta$ of the quadrotor. By choosing a RPY angular representation, $R_{b}^{i}$ becomes:

$$
R_{b}^{i}=\left[\begin{array}{ccc}
C_{\vartheta} C_{\psi} & C_{\vartheta} S_{\psi} & -S_{\vartheta} \\
S_{\varphi} S_{\vartheta} C_{\psi}-C_{\varphi} S_{\psi} & S_{\varphi} S_{\vartheta} S_{\psi}+C_{\varphi} C_{\psi} & S_{\varphi} C_{\vartheta} \\
C_{\varphi} S_{\vartheta} C_{\psi}+S_{\varphi} S_{\psi} & C_{\varphi} S_{\vartheta} S_{\psi}-S_{\varphi} C_{\psi} & C_{\varphi} C_{\vartheta}
\end{array}\right]
$$

The vehicle attitude influences both the forces and momenta that are exchanged with the manipulator at the manipulator base, i.e. the term $R_{b}^{i} R_{m}^{b} f_{m a n}^{m}$ and the control inputs for the translational dynamics of the aerial vehicle $f R_{b}^{i}[0,0,-1]^{T}$ in a non-linear manner. Nevertheless, note that the control input of the translational vehicle dynamics in (2)

$$
f R_{b}^{i}\left[\begin{array}{c}
0 \\
0 \\
-1
\end{array}\right]=\left[\begin{array}{c}
f S_{\vartheta} \\
-f S_{\varphi} C_{\vartheta} \\
-f C_{\varphi} C_{\vartheta}
\end{array}\right]
$$

do not have any dependency on the yaw angle $\psi$. The nonlinear control input can thus be considered as a function of the only thrust force $f$ and on the components $\varphi$ and $\vartheta$ of the attitude $\Theta$ of the vehicle. It is now possible to define a proper control law which exploit the inputs $f, \varphi$ and $\vartheta$ to control the linear dynamics of the quadrotor, and the control input $f_{m a n}^{m}$ as a control input for the manipulator.

\section{B. Passivity-Based Control of the Aerial Manipulator}

The goal of the control law for the approximated aerial manipulator (2) is twofold. On one hand, it has to stabilize a constant free-flight configuration, namely to maintain the end-effector in the desired vertical and lateral positions. On the other hand, it has to dock to a vertical surface by applying a certain force by means of the manipulator's end-effector. Motivated by the effectiveness of the energybased approaches [23] in physical interaction between robots and the environment [24], an impedance controller [25] is proposed. A graphical intuition of the overall passivity-based controller for the aerial manipulator is shown in Fig. 4.

By considering system (2), let the input (3) be

$$
f\left[\begin{array}{c}
S_{\vartheta} \\
-S_{\varphi} C_{\vartheta} \\
-C_{\varphi} C_{\vartheta}
\end{array}\right]=\mathbf{u}+m_{\text {uav }} g \hat{\mathbf{z}}^{i}
$$

where $\mathbf{u}=\left[u_{x}, u_{y}, u_{z}\right]^{T} \in \mathbb{R}^{3}$ is the new input. Note that (4) is well defined for all $\mathbf{u} \in \mathbb{R}^{3}$ so that $\left|\mathbf{u}+m_{\text {uav }} g \hat{\mathbf{z}}^{i}\right|>0$.

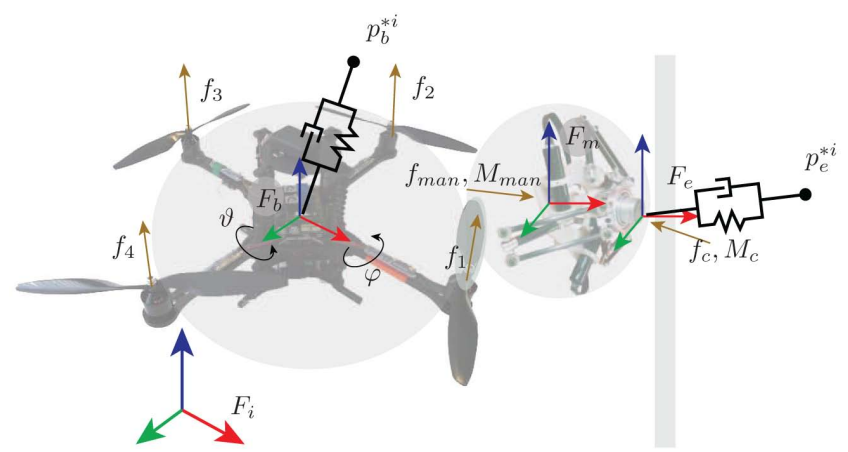

Fig. 4. The passivity-based control architecture for the flying robot.

Accordingly, system (2) can be rewritten as

$$
\begin{aligned}
\dot{p}_{b}^{i} & =\mathbf{v}^{i} \\
m_{u a v} \dot{\mathbf{v}}^{i} & =\mathbf{u}+d(t)
\end{aligned}
$$

in which $d(t):=R_{b}^{i} R_{m}^{b} f_{\text {man }}^{m}(t)$.

Let $p_{b}^{\star} i=\left[x^{\star}, y^{\star}, z^{\star}\right]^{T}$ be the desired constant reference for the lateral, longitudinal and vertical position of the vehicle. Moreover let the control input $\mathbf{u}$ be designed as

$$
\mathbf{u}=-K_{p}\left(p_{b}^{i}-p_{b}^{\star i}\right)-K_{d} \mathbf{v}^{i}
$$

with $K_{p}, K_{d} \in \mathbb{R}^{3 \times 3}$ positive definite matrices. The above linear controller can be interpreted as a passivity-based control law. In fact, the resulting closed loop system

$$
m_{u a v} \dot{\mathbf{v}}^{i}+K_{d} \mathbf{v}^{i}+K_{p}\left(p_{b}^{i}-p_{b}^{\star i}\right)=d(t)
$$

turns out to be output strictly passive by choosing input $d$, output $\mathbf{v}^{i}$ and storage function $V\left(\mathbf{v}^{i}, p_{b}^{i}\right)=\mathcal{K}\left(\mathbf{v}^{i}\right)+\mathcal{P}\left(p_{b}^{i}\right)$, in which $\mathcal{K}\left(\mathbf{v}^{i}\right)=\frac{1}{2} m_{\text {uav }}\left(\mathbf{v}^{i}\right)^{T} \mathbf{v}^{i}$ denotes the kinetic energy and $\mathcal{P}\left(p_{b}^{i}\right)=\frac{1}{2}\left(p_{b}^{i}-p_{b}^{\star i}\right)^{T} K_{p}^{\gamma}\left(p_{b}^{i}-p_{b}^{\star}\right)$ denotes the potential energy that has a minimum in $p_{b}^{\star i}$. As shown in [26][Lemma 6.7], the property of output strict passivity can be linked to zero-input asymptotic stability via zero-state observability. This property holds for the linear dynamics (5).

This shows that in free-flight, when the manipulator is kept at a constant position and the reaction forces are zero, the UAV asymptotically reaches the desired set-points $p_{b}^{\star i}$.

Also in the docking scenario, the manipulator is controlled in order to be passive. By using the passivity properties of the overall closed-loop system (since the environment, the manipulator and the UAV are passive), an impedance controller is implemented [25] to regulate either the applied forces to the environment, or the final equilibrium configuration. This is achieved by properly choosing the desired set-points for both the aerial vehicle and the manipulator, and the stiffness of the passivity-based controllers.

\section{EXPERIMENTAL RESULTS}

In this Section, the experimental results of an interaction task are presented. The experiments aim to show the dynamic behavior of the controlled platform. 


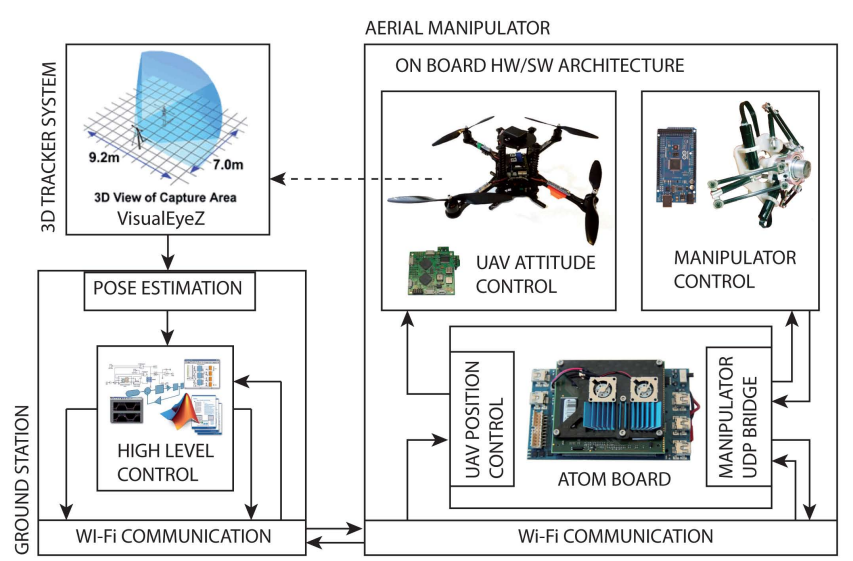

Fig. 5. System overview - The overall system consists of the AscTec Pelican quadrotor and the manipulation system. An external marker tracker is used to estimate the absolute pose of the vehicle. The ground station, allows the intercommunication between the different submodules and devices.

\section{A. System Overview}

The overall hardware and software architecture is depicted in Fig. 5. The UAV is an AscTec Pelican quadrotor [27], whose low level attitude control is performed onboard by two ARM7 micro processors. An additional processor, i.e. an Intel Atom 1.6 GHz processor, performs the position control. The manipulator, mounted on one side of the quadrotor, has 7 DOFs and has been designed for inspection of industrial environments [18]. The mechanics consists of a 3 DoFs cartesian Delta structure and a 4 DoFs end-effector, realized by a Cardan gimbal system. The end-effector has two passive rotational joints (for the pitch and yaw movements), one passive linear joint in the direction of the interaction and one actuated joint for the roll motion (see Figure 6(b)). Four DC motors, equipped with encoders, are used are controlled onboard by an Arduino ATMega2560 with a rate of $2.5 \mathrm{~ms}$.

The experiments have been conducted in an indoor test area. The overall system relies on an external positioning system, i.e., a PTI PhoeniX VisualEyez VZ 4000 unit [28], which captures the motion of the active markers (LED). The absolute position measurements of the markers are used for the estimation of the quadrotor pose, and to stabilize the position of the vehicle. Finally, the trajectory generation and the pose estimation run on the ground station, with an Ubuntu Linux 10.10 operative system. The different software modules communicate over a WiFi data link (802.11n standard).

\section{B. Experiment Description}

With reference to Fig. 3, let the position of the end-effector with reference to the inertial frame be:

$$
p_{e}^{i}=p_{b}^{i}+R_{b}^{i} \bar{p}_{m}^{b}+R_{b}^{i} \bar{R}_{m}^{b} p_{e}^{m}
$$

The system is controlled to realize an interaction of the manipulation system with a vertical wall, as depicted in Fig. 6(a). During the experiment, the flying robot approaches the vertical surface, placed at about $p^{\star}=[1.2,0,0.5]^{T} m$, and realizes a contact with the manipulator's end effector.

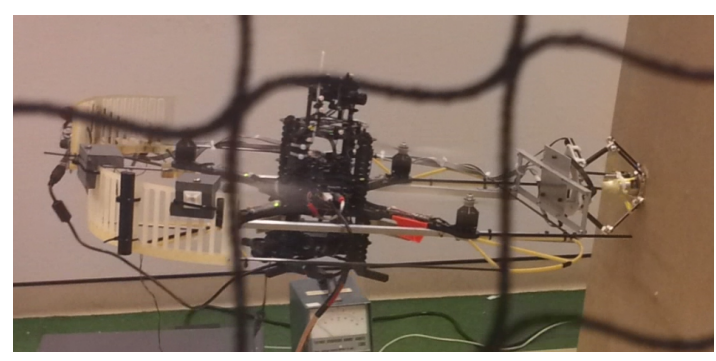

(a)

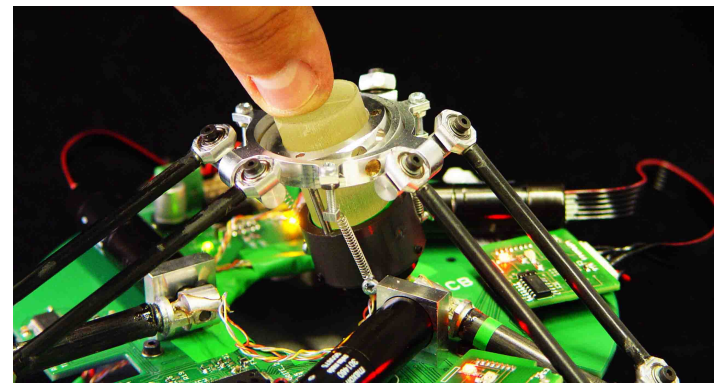

(b)

Fig. 6. 6(a) AscTec Pelican quadrotor during interaction with a wall by means of the manipulation system. 6(b) The manipulation system prototype.
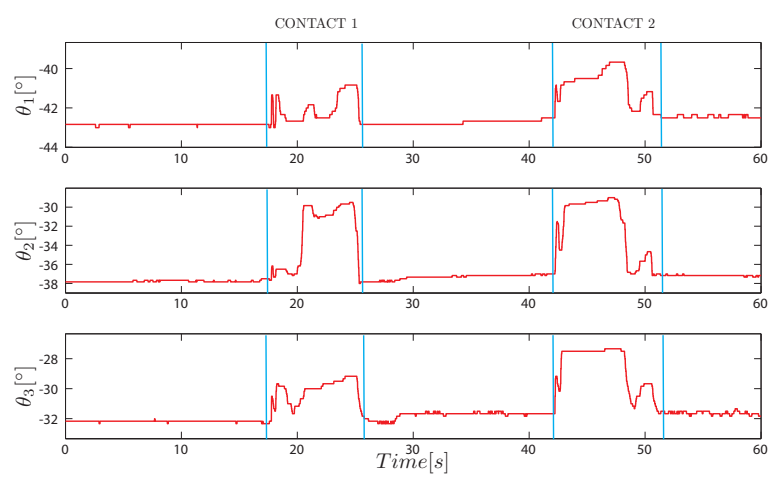

Fig. 7. Joint angles of the Delta manipulator during interaction with a wall. When the interaction occurs, the angles $\theta_{i}$ are deviated from their actual position because of the interaction force.

\section{Experimental Validation}

Since the flying robot is controlled as an impedance, when the interaction with the surface occurs, a displacement at the joints of the manipulator is observed. Fig. 7 shows the three subplots that reports the measurement of the joint angles of the Delta structure at a given configuration of the manipulator, while impacting a wall. The fourth actuated DOF (i.e. the end-effector roll motion) and the passive rotation of the end-effector are not considered here. When the manipulator interacts with the wall, a disturbance on the vehicle is generated. As a consequence the UAV reacts with an actuation force that depends on the tracking error of the UAV trajectory. Nevertheless, in order to generate the lateral actuation force, the system has to tilt (see Fig. 8), as a consequence of the under-actuation of the quadrotor. More specifically, during this experiment, the reference position of the manipulator is fixed with respect to $F_{m}$, i.e. $p_{e}^{m \star}$ is 


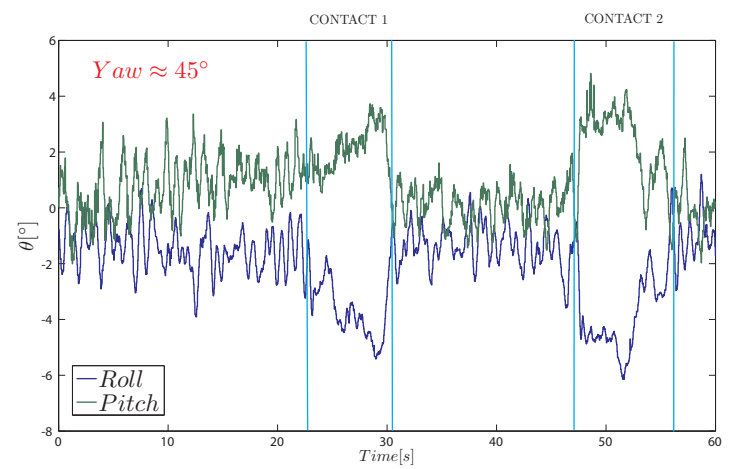

Fig. 8. Measured attitude of the quadrotor at the reference frame $F_{b}$. Note the importance of the attitude regulation in order to guarantee a certain force to contrast the contact force.
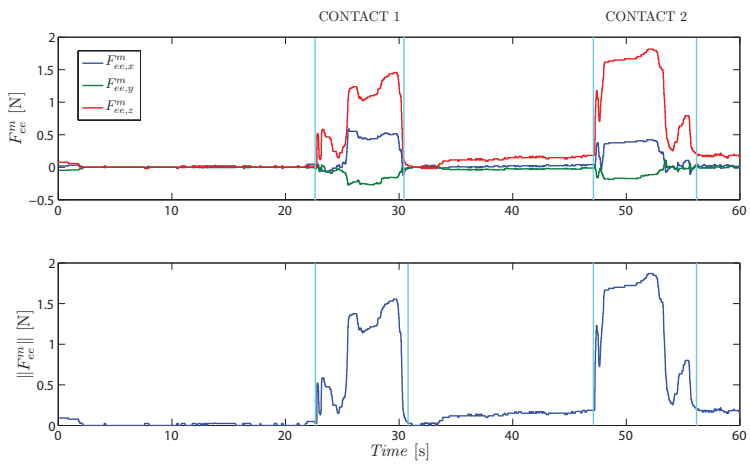

Fig. 9. Estimation of the contact forces during the interaction task. Top: the three components of the estimated external force during the task. Bottom: the norm of the overall force acting at the end-effector.

kept constant. A reference position is set to the quadrotor, such that the system enters in contact with the wall. When the system touches the wall, the manipulator moves as a consequence of the intrinsic compliance of the low-level impedance control. The impedance behavior of the UAV generates a virtual force on the vehicle that depends on its position and velocity error, but in order to generate this force, a pitch and roll displacements are required from the low level control. Fig. 9 show an estimation of the interaction forces with respect to the manipulator frame $F_{m}$. The estimated forces have been computed by using the displacements from the reference position of the joint angles and the knowledge on the system model and of the control gains. Note that the force estimation is not here used for control purposes.

\section{CONCLUSIONS}

In this paper, an interaction control of a flying robot is presented. The mechanics of the system consists on a quadrotor aerial vehicle, which mounts on one side a manipulation system. The purpose of this work has been to study the underactuated manipulation system for inspection through physical interaction. A passivity based approach is exploited to derive a control law that guarantee stability during free flight and interaction. Experimental results shows the effectiveness of the proposed control strategy for the interaction with a remote environment.

\section{REFERENCES}

[1] A. Bachrach, R. He, and N. Roy, "Autonomous flight in unknown indoor environments," International Journal of Micro Air Vehicles, vol. 1, no. 4, pp. 217-228, 2009.

[2] K. Valavanis, Ed., Advances in Unmanned Aerial Vehicles. Springer, 2007.

[3] P. Pounds, R. Mahony, and P. Corke, "Modelling and control of a quad-rotor robot," in Proceedings of the Australasian Conference on Robotics and Automation, 2006.

[4] S. Bouabdallah and R. Siegwart, Advances in Unmanned Aerial Vehicles. Springer, 2007, ch. Chapter 6: Design and Control of a Miniature Quadrotor, pp. 171-210.

[5] V. Gavrilets, "Aerobatic maneuvering of miniature helicopters," Ph.D. Thesis, Massachussetts Institute of Technology, 2003.

[6] J. Pflimlin, P. Soueres, and T. Hamel, "Hovering flight stabilization in wind gusts for ducted fan uav," Proceedings of the IEEE Conference on Decision and Control, 2004.

[7] M. W. Spong, Underactuated mechanical systems. Springer, 1998.

[8] A. Albers, S. Trautmann, T. Howard, T. Nguyen, M. Frietsch, and C.Sauter, "Semi-autonomous flying robot for physical interaction with environment," in Proceedings of the IEEE Conference on Robotics Automation and Mechatronics, 2010.

[9] N. Michael, J. Fink, and V. Kumar, Cooperative manipulation and transportation with aerial robots. Autonomous Robots - Springer, 2010.

[10] Q. Lindsey, D. Mellinger, and V. Kumar, "Construction of cubic structures with quadrotor teams," in Proceedings of Robotics: Science and Systems, 2011.

[11] A. Desbiens and M. Cutkosky, "Landing and perching on vertical surfaces with microspines for small unmanned air vehicle," Journal of Intelligent \& Robotic Systems, vol. 1, no. 4, pp. 313-327, 2009.

[12] A. Frank, J. McGrew, M. Valenti, D. Levine, and J. How, "Hover, transition, and level flight control design for a single-propeller indoor airplane," in Proceedings of the AIAA Guidance, Navigation and Control Conference, 2007.

[13] P. Pounds, D. Bersak, and A. Dollar, "Grasping from the air: Hovering capture and load stability," in Proceedings of the IEEE International Conference on Robotics and Automation, 2011.

[14] C. Korpela, T. Danko, and P. Oh, "MM-UAV: Mobile manipulating unmanned aerial vehicle," Journal of Intelligent \& Robotic Systems, pp. 1-9, Nov. 2011.

[15] AIRobots, http://www.airobots.eu.

[16] M. Reyhanoglu, A. van der Schaft, N. McClamroch, and I. Kolmanovsky, "Dynamics and control of a class of underactuated mechanical systems," IEEE Transactions on Automatic Control, vol. 44 no. 9, pp. 1663 - 1671, 1999.

[17] R. Olfati-Saber, "Nonlinear control of underactuated mechanical systems with application to robotics and aerospace vehicles," Ph.D. Thesis, Massachussetts Institute of Technology, 2001.

[18] A. Keemink, M. Fumagalli, S. Stramigioli, and R. Carloni, "Mechanical design of a manipulation system for unmanned aerial vehicles," in Proceedings of the IEEE International Conference on Robotics and Automation, 2012.

[19] A. Tayebi and S. McGilvray, "Attitude stabilization of a vtol quadrotor aircraft," IEEE Transactions Control Systems Technology, vol. 14, pp. 562-571, 2006.

[20] N. Diolaiti, C. Melchiorri, and S. Stramigioli, "Contact impedance estimation for robotic systems," IEEE Transactions on Robotics, vol. 21, no. 5, pp. 925-935, 2005.

[21] R. Murray, A mathematical introduction to robotic manipulation. CRC Press, 1994

[22] A. Isidori, Nonlinear Control Systems II, ser. Communication and Control Engineering Series. Springer-Verlag London, 1998.

[23] R. Ortega, A. van der Schaft, I. Mareels, and B. Maschke, "Putting energy back in control," IEEE Control Systems Magazine, vol. 21, no. 2, pp. 18-33, 2001

[24] B. Brogliato, Nonsmooth Mechanics Model, Dynamics and Control. Springer, 1996.

[25] N. Hogan, "Impedance control: an approach to manipulation: parts I-III," ASME Journal o Dynamic Systems, Measurement and Control, vol. 107 , no. 1 , pp. 1-24, 1985.

[26] H. K. Khalil, Nonlinear systems. Prentice Hall, 1996.

[27] Ascending Technologies GmbH (AscTec), http://www.asctec.de.

[28] PTI PhoeniX, "VisualEyez VZ4000," http://www.ptiphoenix.com. 